[2] Eder L, Thavaneswaran A, Chandran V, Cook R, Gladman DD. Increased burden of inflammation over time is associated with the extent of atherosclerotic plaques in patients with psoriatic arthritis. Ann Rheum Dis. 2015 Oct;74(10):1830-5.

[3] Shang Q, Tam LS, Sanderson JE, Lee AP, Li EK, Yu CM. Impaired left ventricular apical rotation is associated with disease activity of psoriatic arthritis. J Rheumatol. 2014 Apr;41(4):706-13.

[4] Blessberger $\mathrm{H}$, Binder T. NON-invasive imaging: Two dimensional speckle tracking echocardiography: basic principles. Heart. 2010 May;96(9):716-22.

Disclosure of Interests: None declared

DOI: 10.1136/annrheumdis-2019-eular.7909

\section{SAT0386 1 PREVALENCE OF SUBCLINICAL CARDIOVASCULAR DISEASE IN PSORIATIC ARTHRITIS: A MULTICENTRIC STUDY}

Maria Paz Martínez-Vidal ${ }^{1}$, José Andrés Lorenzo Martín ${ }^{2}$, Mariano Andres $^{1}$, Vega Jovani ${ }^{1}$, Carlos Santos Ramirez ${ }^{3}$, Cintia Romera Lopez ${ }^{4}$, Cristina Fernández-Carballido ${ }^{5}$, Rubén Queiró Silva ${ }^{2} .{ }^{1}$ Hospital General

Universitario Alicante, Alicante, Spain; ${ }^{2}$ Hospital Central Universitario Asturias, Oviedo, Spain; ${ }^{3}$ Hospital Virgen de los lirios, Alcoy, Spain; ${ }^{4}$ Hospital del Vinalopó, Elche, Spain; ${ }^{5}$ Hospital Universitario San Juan, Alicante, Spain

Background: There are scarce data regarding subclinical vascular disease in psoriatic arthritis $(P S A)(1)$. Similarly to rheumatoid arthritis, the real CVR could also be underestimated by clinical risk charts (2).

Objectives: To study the prevalence of subclinical vascular atheromatosis in patients with PsA; to analyze the association of carotid plaques with PsA characteristics; to identify which patients with intermediate or high clinical risk would be worthwhile to undergo carotid ultrasound to detect plaque.

Methods: Descriptive, transversal and multicentric study, of patients with clinical diagnose of PsA followed in 4 Spanish hospitals. Demographic data, classic CVR factors and clinical characteristics were recorded. The probability of a 10 years fatal cardiovascular event was calculated by SCORE charts adapted for Spain (3), and afterwards a systematic bilateral common carotid ultrasound (4) was conducted. The intimamedia thickness of the carotid wall and the presence of atheroma plaque were registered. Patient's characteristics were described using descriptive analysis techniques. For univariate comparisons we used Chi2 and Anova. Multivariate analyses were conducted by logistic regression. The probability of plaque according to the age and the presence of CVR factors was calculated for patients with clinical intermediate or high risk by means of logistic regression. The study was approved by the Hospital General Universitario de Elda Ethic Committee (RCV-PS-MC/26/02/2016).

Results: 309 patients were included. The characteristics of the sample and the prevalence of CVR factors are depicted in Table 1. Patients were stratified by SCORE as follows: low risk 41 patients (13.3\%), intermediate 182 (58.9\%), high 75 (24.3\%) and very high 11 (3.6\%)

Table 1.. Clinical characteristics

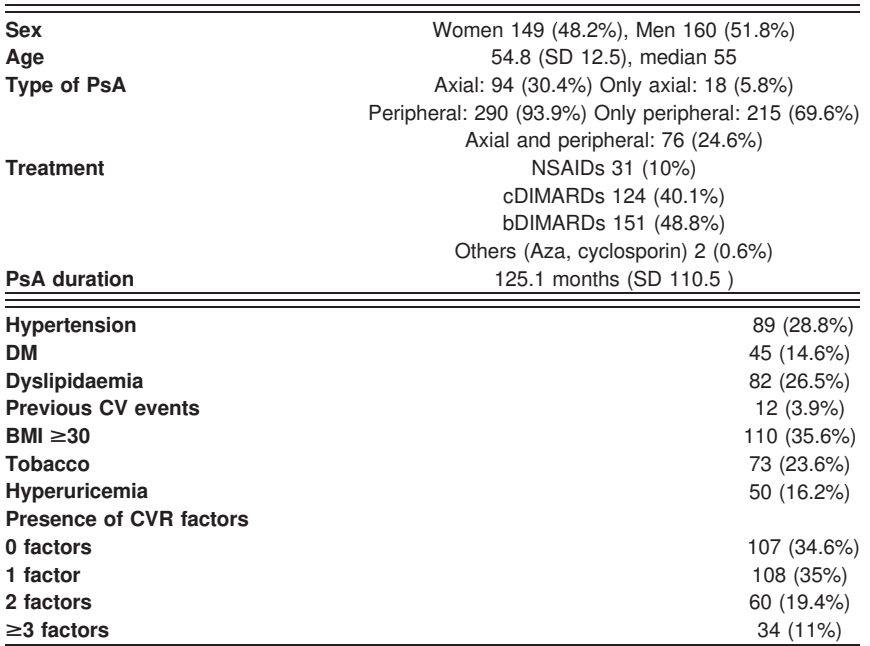

patients. Plaque criteria by ultrasound was found in 95 patients $(30.7 \%)$, who were reclassified as very high risk. Final distribution after carotid ultrasound showed a decrease in all groups $(12 \%, 43.7 \%$ and $14.2 \%$ in low, intermediate and high risk, respectively) with a consequent increase in the very high risk group $(30.1 \%)$. In the univariate study, plaque was associated with age $(p<0.001)$, diabetes $(p=0.004)$, hypertension $(p=0.001)$, hyperuricemia $(p=0.01)$ and dyslipidaemia $(p<0.001)$; any association with PsA characteristics wasn't found. In the multivariate analyses, plaque was associated with age $>55$ years (OR 2.84; IC 1.54-5.55), and with the number of classical CVR factors (OR 1.49; IC 1.13-1.97). In the presence of 3 or more risk factors, the probability of having plaque was $57.2 \%$ in patients with clinical intermediate risk and $61.8 \%$ in patients with high risk, and raised with increasing presence of CVR factors (Table 2).

Conclusion: Subclinical vascular disease is present in almost a third of the patients with PSA. SCORE tables may prove inaccurate for estimating the real CVR, and carotid ultrasound would be advisable in patients with intermediate or high risk who present any classical CVR factor.

Disclosure of Interests: None declared

DOI: 10.1136/annrheumdis-2019-eular.1054

\section{SAT0387 $\quad$ CONSISTENT EFFICACY IN PATIENT SUBGROUPS ACROSS BASELINE DEMOGRAPHICS AND DISEASE CHARACTERISTICS: RESULTS FROM A PHASE 2 STUDY OF GUSELKUMAB IN PATIENTS WITH ACTIVE PSORIATIC ARTHRITIS}

lain McInnes ${ }^{1}$, Philip Helliwell ${ }^{2}$, Alice B. Gottlieb ${ }^{3}$, Wolf-Henning Boehncke ${ }^{4}$, Xie L. Xu ${ }^{5}$, Stephen $\mathrm{Xu}^{5}$, Yuhua Wang ${ }^{5}$, Elizabeth C. Hsia ${ }^{5,6}$, Atul Deodhar ${ }^{7}$.

${ }^{1}$ University of Glasgow, Glasgow, United Kingdom; ${ }^{2}$ Leeds Institute of Rheumatic and Musculoskeletal Medicine, University of Leeds, Leeds, United Kingdom; ${ }^{3}$ Icahn School of Medicine at Mt Sinai, New York, United States of America; ${ }^{4}$ Division of Dermatology and Venereology, Geneva Univ Hospitals and Department of Pathology and Immunology, Univ of Geneva, Geneva, Switzerland; ${ }^{5} \mathrm{Janssen}$ Research and Development, LLC, Spring House, United States of America; ${ }^{6}$ Univ of Pennsylvania, Phila, United States of America; ${ }^{7}$ Oregon Health and Science $U$, Portland, United States of America

Background: Guselkumab (GUS) is a monoclonal antibody targeting interleukin-23 that has demonstrated efficacy in a phase 2 trial of psoriatic arthritis (PsA).

Objectives: Here subgroup analyses were conducted to evaluate the consistency of efficacy on the primary endpoint, ACR20 response.

Methods: In this randomized, double-blind, placebo-controlled, Phase-2 trial, pts with active PsA $(\geq 3$ tender, $\geq 3$ swollen joints, C-reactive protein $>3 \mathrm{mg} / \mathrm{L},>3 \%$ body surface area [BSA] of plaque psoriasis) despite current or previous treatment with standard-of-care therapies were randomized 2:1 to subcutaneous GUS $100 \mathrm{mg}(\mathrm{n}=100)$ or placebo (PBO, $n=49$ ) at Wk0, Wk4, and every 8 wks (q8w) through Wk44. At Wk16, pts with $<5 \%$ improvement in both swollen and tender joints could escape early to open-label ustekinumab. Pts continuing PBO crossed-over to receive GUS $100 \mathrm{mg}$ at Wk 24, 28 then q8w through Wk44. The primary analysis was performed in a modified Intent-to-Treat (mITT) population which include all randomized subjects who received at least 1 administration of study agent based on their assigned treatment regardless actual treatment received. Pts who met treatment failure criteria, escaped early, or had missing data at Wk24 were considered non-responders for ACR20 at Wk24. Pre-planned subgroup analyses by demographic and disease characteristics at baseline and PsA medication use were performed, using the same data handling rules as in the primary analysis.

Results: At Wk24, 58/100 (58.0\%) of pts in the GUS vs $9 / 49(18.4 \%)$ in the PBO group achieved an ACR20 response $(p<0.001)$. Efficacy was consistently observed in subgroups defined by demographics (gender, age, weight, region), disease characteristics at baseline (disease duration, PsA subtypes, tender/swollen joint counts, HAQ-DI, CRP, presence of dactylitis or enthesitis, PASI, and BSA) or PsA medication use (prior use of DMARDs or anti-TNFs, concomitant use of MTX, oral corticosteroids, or NSAIDs) (Table). The treatment effect was statistically significant in the majority of subgroups with the lower bound of the 95\% confidence interval of the difference between GUS and PBO exceeding 0 in favor of GUS. There are a few exceptions where small sample sizes of the subgroups limited the interpretation. All enrolled subjects were white; therefore, the effect of guselkumab in other racial groups could not be assessed. Among GUS-treated pts, pts with enthesitis, dactylitis, PASI $\geq 12$, BSA $>10 \%$, tender joint count $\leq 10$ or swollen joint count $\leq 10$ appeared to have numerically higher ACR20 response rate than those corresponding subgroups defined otherwise, although the sample size is small and the data should be interpreted with caution. 


\begin{tabular}{|c|c|c|c|}
\hline \multicolumn{4}{|c|}{$\begin{array}{l}\text { Table. Number of Patients Achieving ACR-20 Response at Week } 24 \text { by Treatment Group in } \\
\text { Subgroups of Patients Defined by Baseline Demographic/Disease Characteristics and PSA } \\
\text { Medication Use }\end{array}$} \\
\hline & $\begin{array}{l}\text { Placebo } \\
\mathrm{n} / \mathrm{N}(\%)\end{array}$ & $\begin{array}{c}\text { Guselkumab } \\
\mathrm{n} / \mathrm{N}(\%)\end{array}$ & Difference $(95 \% \mathrm{CI})$ \\
\hline All Patients & $9 / 49(18.4)$ & $58 / 100(58.0)$ & $39.6(25.2,54.1)$ \\
\hline Gender Male & $5 / 24(20.8)$ & $29 / 52(55.8)$ & $33.8(12.6,54.9)$ \\
\hline \multirow{2}{*}{$\begin{array}{lr} & \text { remale } \\
\text { Age (yrs) } & <65 \\
265\end{array}$} & $\frac{4 / 25(16.0)}{9 / 47(19.1)}$ & $\frac{29 / 48(60.4)}{48 / 89(53.9)}$ & $\frac{43.2(22.8,63.7)}{34.7(19.6,49.9)}$ \\
\hline & $\begin{array}{l}9 / 21(0) \\
0 / 2(1)\end{array}$ & $10 / 11(90.9)$ & $90.9(73.9,100.0)$ \\
\hline $\begin{array}{ll}\text { Weight (kg) } \quad 590 \\
90\end{array}$ & $\begin{array}{l}8 / 31(25.8) \\
1 / 18(5.6)\end{array}$ & $\begin{array}{l}38 / 67(56.7) \\
30 / 3360.6)\end{array}$ & $31.2(11.9,50.5)$ \\
\hline $\begin{array}{l}\text { USA, Canada, Spain, or Germany } \\
\text { Poland, Russia, or Romania }\end{array}$ & $\begin{array}{l}2 / 11(18.2) \\
7 / 38(18.4)\end{array}$ & $\begin{array}{l}9 / 19(47.4) \\
49 / 81(60.5)\end{array}$ & $\begin{array}{l}29.9(0.0,59.7) \\
42.4(26.2,58.5)\end{array}$ \\
\hline (yrs) $\quad \begin{aligned} & 1 \text { to }<3 \\
& \quad 23 \\
&\end{aligned}$ & $\begin{array}{l}1 / 10(10.0) \\
5 / 10(50.0) \\
3 / 29(10.3)\end{array}$ & $\begin{array}{l}10 / 15(66.7) \\
16 / 25(64.0) \\
32 / 60(53.3)\end{array}$ & $\begin{array}{l}56.7(26.4,86.9) \\
16.7(-19.6,52.9) \\
43.0(26.4,59.6)\end{array}$ \\
\hline \multirow[t]{2}{*}{$\begin{array}{l}\text { Arthritis Mutilans } \\
\text { Asymmetric Peripheral Arthritis } \\
\text { Dip Joint Involvement } \\
\text { Polyarticular Arthritis with } \\
\text { Absence of Rheumatoid Nodules }\end{array}$} & $\begin{array}{l}0 / 3(0) \\
2 / 14(14.3) \\
3 / 7(42.9)\end{array}$ & $\begin{array}{c}0 \\
18 / 26(69.2) \\
8 / 17(47.1)\end{array}$ & $\begin{array}{l}52.3(25.4,79.2) \\
-2.9(-49.5,43.6)\end{array}$ \\
\hline & $\begin{array}{r}3 / 20(15.0) \\
1 / 5(20.0)\end{array}$ & $\begin{array}{l}19 / 39(48.7) \\
13 / 18(72.2)\end{array}$ & $\begin{array}{l}32.1(9.1,55.0) \\
48.8(7.0,90.5)\end{array}$ \\
\hline Bpollen joints $<5$ & $\begin{array}{c}1 / 7(0.0) \\
0 / 7(0) \\
5 / 25(20.0)\end{array}$ & $\begin{array}{l}4 / 10(40.0) \\
28 / 44(63.6)\end{array}$ & $\begin{array}{l}40.0(9.6,70.4) \\
43.1(22.0,64.2)\end{array}$ \\
\hline $\begin{array}{r}5-10 \\
>10\end{array}$ & $4 / 17(23.5)$ & $26 / 46(56.5)$ & \\
\hline $\begin{array}{r}5-10 \\
>10\end{array}$ & $\begin{array}{l}0 / 1(0) \\
0 / 11(0) \\
9 / 37(24.3)\end{array}$ & $\begin{array}{l}1 / 2(50.0) \\
13 / 20(65.0) \\
44 / 78(56.4)\end{array}$ & $\begin{array}{l}50.0(-19.3,100.0) \\
66.6(46.5,86.8) \\
32.1(14.7,49.6)\end{array}$ \\
\hline $\begin{array}{ll}\text { CRP } & <1 \\
\geq 1\end{array}$ & $\begin{array}{l}5 / 28(17.9) \\
4 / 21(19.0)\end{array}$ & $\begin{array}{l}29 / 53(54.7) \\
29 / 47(61.7)\end{array}$ & $\begin{array}{l}36.5(16.9,56.1) \\
41.9(20.163 .6)\end{array}$ \\
\hline HAQ-DI & $\begin{array}{l}4 / 21(10.0) \\
1 / 10(10.0) \\
8 / 39(20.5)\end{array}$ & $\begin{array}{l}21 / 19(57.9) \\
47 / 81(58.0)\end{array}$ & $\begin{array}{l}41.5(2.1 .1,03.0) \\
46.8(16.5,77.0) \\
37.5(21.0,53.9)\end{array}$ \\
\hline $\begin{array}{ll}\text { PASI Score } \quad<12 \\
\end{array}$ & $\begin{array}{l}6 / 31(19.4) \\
0 / 9(0) \\
2 / 8(25.0)\end{array}$ & $\begin{array}{l}30 / 60(50.0) \\
12 / 17(70.6) \\
15 / 21(71.4)\end{array}$ & $\begin{array}{l}30.9(12.2,49.6) \\
72.4(52.7,92.2) \\
43.4(6.9,80.0)\end{array}$ \\
\hline $\begin{array}{lr}\text { Psoriatic BSA } \begin{array}{r}3-10 \\
(\%) \\
10-20\end{array}\end{array}$ & $\begin{array}{l}6 / 29(20.7) \\
1 / 6(16.7) \\
2 / 14(143)\end{array}$ & $\begin{array}{l}24 / 43(55.8) \\
17 / 30(56.7) \\
17 / 27(63.0)\end{array}$ & $\begin{array}{l}34.9(14.0,55.9) \\
40.5(5.5,75.5) \\
48.1(22.37 .9)\end{array}$ \\
\hline $\begin{array}{ll}\text { Enthesitis } & \text { Yes }\end{array}$ & $\begin{array}{l}5 / 31(16.1) \\
4 / 18(22.2)\end{array}$ & $\begin{array}{l}46 / 76(60.5) \\
12 / 24(50.0)\end{array}$ & $\begin{array}{l}43.9(27.1,60.8) \\
26.7(-0.2,53.5)\end{array}$ \\
\hline Dactylitis & $\begin{array}{l}4 / 10(2.2) \\
6 / 23(26.1) \\
3 / 26(11.5)\end{array}$ & $\begin{array}{l}12 / 54(50.0) \\
22 / 42(52.1) \\
22.4)\end{array}$ & $\begin{array}{l}35.8(14.1,57.5) \\
40.8(21.5,60.2)\end{array}$ \\
\hline Prior PsA Med use: & & & \\
\hline $\begin{array}{cc} & \text { No } \\
\text { DMARD } & 0 \\
& 1\end{array}$ & $\begin{array}{l}0 / 4(0) \\
9 / 45(20.0) \\
2 / 8(25.0) \\
5 / 33(15.2) \\
2 / 8(25.0)\end{array}$ & $\begin{array}{c}6 / 9(66.7) \\
52 / 91(57.1) \\
4 / 10(40.0) \\
43 / 71(60.6) \\
11 / 19(57.9)\end{array}$ & $\begin{array}{l}66.7(35.9,97.5) \\
37.1(21.7,52.6) \\
15.0(-27.7,57.7) \\
45.4(28.8,62.0) \\
30.6(-7.2,68.3)\end{array}$ \\
\hline Baseline PsA Med use: & & & \\
\hline \multirow{2}{*}{ 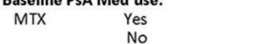 } & $5 / 19(26.3)$ & $27 / 47(57.4)$ & $30.0(5.8,54.2)$ \\
\hline & & $31 / 53(58$ & $44.4(26.5,62.2)$ \\
\hline \multirow{2}{*}{$\begin{array}{l}\text { Corticosteroid Yes } \\
\text { (oral) No }\end{array}$} & $2 / 8(25.0)$ & $10 / 12(83.3)$ & $61.3(27.3,95.4)$ \\
\hline & $7 / 41(17.1)$ & $48 / 88(54.5)$ & $37.5(22.0,52.9)$ \\
\hline NSAID Yes & $7 / 36(19.4)$ & $42 / 70(60.0)$ & $40.6(23.5,57.8)$ \\
\hline No & $2 / 13(15.4)$ & $16 / 30(53.3)$ & $38.3(12.0,64.6)$ \\
\hline $\begin{array}{l}\text { ACR-20 = } 20 \% \text { improvement in Am } \\
\text { confidence interval; CRP = C-Reacti } \\
\text { Index; MTX = methotrexate; Med= } \\
\text { Psoriasis Area and Severity Index; } \\
95 \% \text { CI's were calculated based on }\end{array}$ & $\begin{array}{l}\text { ic arthrit } \\
\text { ics. }\end{array}$ & ssment $C$ & $\begin{array}{l}\text { Y Surface Area; } \mathrm{Cl}= \\
\text { naire-Disability } \\
\text { pry drug; PASI = }\end{array}$ \\
\hline
\end{tabular}

Conclusion: In this phase 2 trial of patients with PsA, GUS demonstrated efficacy consistently across subgroups of pts according to baseline demographics, disease characteristics, and PsA-medication use. The relationship between baseline characteristics and clinical outcome will be further explored in phase 3 studies with a larger PsA population.

\section{REFERENCE}

[1] Deodhar A, et al. Lancet 2018;391: 2213-24.

Disclosure of Interests: lain Mclnnes Grant/research support from: AstraZeneca, Celgene, Compugen, Novartis, Roche, UCB Pharma, Consultant for: AbbVie, Celgene, Galvani, Lilly, Novartis, Pfizer, UCB Pharma, Philip Helliwell Grant/research support from: Paid to charity: from AbbVie, Janssen and Novartis, Consultant for: Paid to charity: from AbbVie, Amgen, Pfizer, and UCB and Celgene. Paid to self: from Celgene and Galapagos, Alice B Gottlieb Grant/research support from: PI: Incyte Corporation, Janssen-Ortho Inc., Lilly ICOS LLC, Novartis, UCB, XBiotech, Consultant for: AbbVie, Dermira, Incyte Corporation, Lilly ICOS LLC, Novartis, Sun Pharmaceutical Industries Ltd., Avotres (unpaid), XBiotech (unpaid), Speakers bureau: AbbVie, Eli Lilly and Company, Janssen Biotech; advisory board: Bristol-Myers Squibb, Celgene Corporation, Janssen Biotech, Janssen-Ortho Inc., LEO Pharma, Novartis, UCB, Wolf-Henning Boehncke Consultant for: Pfizer Inc, Speakers bureau: Pfizer Inc, Xie L Xu Employee of: Employee of Janssen Research \& Development, LLC, Stephen Xu Employee of: Employee of Janssen Research \& Development, LLC, Yuhua Wang Employee of: Employee of Janssen Research \& Development, LLC, Elizabeth C Hsia Employee of: Employee of Janssen Research \& Development, LLC, Atul Deodhar Grant/research support from: AbbVie, Amgen, Eli Lilly, GSK, Janssen, Novartis, Pfizer, and UCB, Consultant for: AbbVie, Amgen, BMS, Eli Lilly, Janssen, Novartis, Pfizer, and UCB

DOI: 10.1136/annrheumdis-2019-eular.1995

\section{SAT0388 CHARACTERIZATION OF PATIENTS WITH PSORIATIC ARTHRITIS AND NAIL PSORIASIS: DATA FROM THE CORRONA PSORIATIC ARTHRITIS/ SPONDYLOARTHRITIS (PSA/SPA) REGISTRY}

Philip J. Mease ${ }^{1}$, Peter Hur ${ }^{2}$, Mei Liu ${ }^{3}$, Sabrina Rebello ${ }^{3}$, Robert Mclean ${ }^{3}$, Blessing Dube ${ }^{3}$, Meghan Glynn ${ }^{3}$, Alexis Ogdie ${ }^{4}{ }^{1}$ Swedish Medical Center/ Providence St. Joseph Health and University of Washington, Seattle, United States of America; ${ }^{2}$ Novartis Pharmaceuticals Corporation, East Hanover, United States of America; ${ }^{3}$ Corrona, LLC, Waltham, United States of America; ${ }^{4}$ Perelman School of Medicine at the University of Pennsylvania, Philadelphia, United States of America

Background: Nail disease is an important feature of PsA and may even precede the disease by many years. ${ }^{1}$ Nail psoriasis is considered one of the 6 disease domains of $\mathrm{PsA}^{2}$; however, there have been limited realworld studies that have examined characteristics of patients with PsA and nail psoriasis, particularly in the United States.

Objectives: To characterize the disease activity, quality of life, and work productivity of patients with PsA with and without nail psoriasis in the US-based Corrona PsA/SpA Registry.

Methods: This study included all patients in the Corrona PsA/SpA registry enrolled between March 2013 and October 2018 with a diagnosis of PsA who had non-missing data on physician-reported nail psoriasis. Patients were stratified by presence vs absence of nail psoriasis at the time of enrollment, defined as a non-zero response on the nail psoriasis visual analog scale (VAS) of $0-100$. Descriptive analyses of patient demographics, disease activity, quality of life, and work productivity were assessed at enrollment and compared between nail psoriasis groups using $t$-tests or Wilcoxon rank-sum tests for continuous variables and $\chi^{2}$ or Fisher's exact tests for categorical variables.

Results: A total of 2841 patients with PsA met the inclusion criteria, including 1152 patients (40.5\%) with nail psoriasis and 1689 patients $(59.5 \%)$ without nail psoriasis at enrollment. Patients with and without nail psoriasis were similar in terms of demographic and clinical characteristics; however, patients with nail psoriasis were slightly younger (53.1 vs 54.4 years) and more likely to be male $(51.9 \%$ vs $44.1 \%)$ and have a higher history of depression (17.8\% vs $13.3 \%$ ) compared with patients without nail psoriasis (all $P<0.05$ ). Patients with nail psoriasis had more severe psoriasis and PsA disease activity, as reflected by statistically significantly higher percentage of affected body surface area (BSA), higher tender and swollen joint counts, decreased likelihood of being in minimal disease activity, worse mean Disease Activity in Psoriatic Arthritis (DAPSA) and Psoriatic Arthritis Disease Activity Score (PASDAS) values, and increased likelihood of having enthesitis and dactylitis at enrollment compared with patients without nail psoriasis (Table 1; all $P<0.05$ ). Patients with nail psoriasis had worse self-reported physical function (HAQ-DI), pain, fatigue, EQ-5D scores, and work and activity impairment compared with patients without nail psoriasis (Table 2; all $P<0.05$ ).

Conclusion: PsA patients with nail psoriasis at the time of registry enrollment had worse disease activity, quality of life, and work productivity compared with those patients without nail involvement. These findings emphasize the importance of identification and management of nail disease in patients with PsA.

\section{REFERENCES}

[1] Gladman DD, et al. Ann Rheum Dis. 2005;64(suppl 2):ii14-7.

[2] Coates LC, et al. Arthritis Rheumatol. 2016;68(5):1060-71.

Acknowledgement: This study was sponsored by Corrona, LLC. Corrona is supported through contracted subscriptions with multiple pharmaceutical companies. The abstract was a collaborative effort between Corrona and Novartis, with financial support provided by Novartis.

Disclosure of Interests: Philip J Mease Grant/research support from: AbbVie, Amgen, BMS, Celgene, Janssen, Lilly, Novartis, Pfizer, SUN and UCB, Consultant for: AbbVie, Amgen, BMS, Galapagos, Gilead Sciences, Inc., Janssen, Lilly, Novartis, Pfizer, SUN and UCB, Speakers bureau: AbbVie, Amgen, BMS, Celgene, Genentech, Janssen, Lilly, Novartis, Pfizer and UCB, Peter Hur Employee of: Peter Hur is an employee of Novartis Pharmaceuticals Corporation, East Hanover, New Jersey, USA, Mei Liu Employee of: M. Liu is an employee of Corrona, LLC., Sabrina Rebello Employee of: Corrona, LLC, Robert McLean: 
Table 1. Disease Activity Among Patients With PsA at Enrollment Stratified by Presence of Nail Psoriasis

\begin{tabular}{|c|c|c|c|}
\hline Characteristic* & $\begin{array}{l}\text { With Nail PsO } \\
\quad(\mathrm{N}=1152)\end{array}$ & $\begin{array}{c}\text { Without Nail } \\
\text { PsO } \\
(\mathrm{N}=1689)\end{array}$ & $P$ Value $^{\dagger}$ \\
\hline Nail PsO (VAS 0-100) & $18.9(22.5)[1152]$ & $0[1689]$ & - \\
\hline Tender joint count $(0-68)$ & $6.0(9.9)[1127]$ & $3.5(7.1)[1639]$ & $<0.01$ \\
\hline Swollen joint count (0-66) & $2.4(4.4)[1125]$ & $1.7(3.5)[1639]$ & $<0.01$ \\
\hline $\mathrm{MDA}, \mathrm{n} / \mathrm{m}(\%)^{ \pm}$ & $359 / 1027(35.0)$ & $716 / 1503(47.6)$ & $<0.01$ \\
\hline DAPSA & $18.1(15.7)[643]$ & $13.4(12.4)[994]$ & $<0.01$ \\
\hline DAPSA group, $n(\%)$ & $n=643$ & $n=994$ & $<0.01$ \\
\hline Remission & $90(14.0)$ & $232(23.3)$ & \\
\hline Low & $231(35.9)$ & $402(40.4)$ & \\
\hline Moderate & $195(30.3)$ & $247(24.8)$ & \\
\hline High & $127(19.8)$ & $113(11.4)$ & \\
\hline PASDAS & $3.8(1.6)[619]$ & $3.3(1.5)[972]$ & $<0.01$ \\
\hline PASDAS group, $n(\%)$ & $n=619$ & $n=972$ & $<0.01$ \\
\hline Inactive & $215(34.7)$ & $465(47.8)$ & \\
\hline Moderate & $132(21.3)$ & $227(23.4)$ & \\
\hline Active & $228(36.8)$ & $239(24.6)$ & \\
\hline Very active & $44(7.1)$ & $41(4.2)$ & \\
\hline Enthesitis, n/m (\%) & $319 / 1152(27.7)$ & $287 / 1689(17.0)$ & $<0.01$ \\
\hline SPARCC Enthesitis Index (1-16) & $3.8(2.9)[319]$ & $3.5(2.8)[287]$ & 0.14 \\
\hline Dactylitis, $\mathrm{n} / \mathrm{m}(\%)$ & $140 / 1152(12.2)$ & $125 / 1689(7.4)$ & $<0.01$ \\
\hline Dactylitis count (1-20) & $2.4(2.3)[140]$ & $2.2(1.8)[125]$ & 0.50 \\
\hline BSA, \% affected & $7.9(14.8)[1125]$ & $3.5(7.6)[1636]$ & $<0.01$ \\
\hline BSA (categorical), n (\%) & $n=1125$ & $n=1636$ & $<0.01$ \\
\hline No disease $(0 \%)$ & $201(17.9)$ & $583(35.6)$ & \\
\hline Mild disease $(>0 \%$ to $\leq 3 \%)$ & $469(41.7)$ & $680(41.6)$ & \\
\hline Moderate disease $>3 \%$ to $\leq 10 \%)$ & $258(22.9)$ & $254(15.5)$ & \\
\hline Severe disease $(>10 \%)$ & $197(17.5)$ & $119(7.3)$ & \\
\hline \multicolumn{4}{|c|}{$\begin{array}{l}\text { BSA, body surface area; DAPSA, Disease Activity in Psoriatic Arthritis; MDA, minimal disease activity; } \\
\text { PASDAS, Psoriatic Arthritis Disease Activity Score; PsO, psoriasis; SPARCC, Spondyloarthritis Research } \\
\text { Consortium of Canada; VAS, visual analog scale. } \\
\text {-All values were calculated based on available data and are presented as "mean (SD) [n]" unless otherwise } \\
\text { stated. } \\
+t \text {-test or Wilcoxon rank-sum test for continuous variables and } x^{2} \text { or Fisher's exact test for categorical } \\
\text { variables. } \\
\text { tMDA is defined as 'yes' if a patient met at least } 5 \text { of the } 7 \text { following categories: tender joint count } \leq 1 \text {, } \\
\text { swollen joint count } \leq 1 \text {, body surface area } \leq 3 \% \text {, patient pain VAS } \leq 15 \text {, patient global activity visual } \\
\text { analogue scale } \leq 20 \text {, health assessment questionnaire } \leq 0.5 \text {, and tender entheseal points } \leq 1 \text {. }\end{array}$} \\
\hline
\end{tabular}

Table 2. Patient-Reported Outcome Measures Among Patients With PsA at Enrollment Stratified by Presence of Nail Psoriasis

\begin{tabular}{|c|c|c|c|}
\hline Characteristic* & $\begin{array}{l}\text { With Nail PsO } \\
(\mathrm{N}=1152)\end{array}$ & $\begin{array}{c}\text { Without Nail } \\
\text { PsO } \\
(\mathrm{N}=1689)\end{array}$ & $P$ Value $^{\dagger}$ \\
\hline Patient pain (VAS 0-100) & $41.7(30.1)[1081]$ & $36.1(29.1)[1603]$ & $<0.01$ \\
\hline Patient-reported fatigue (VAS 0-100) & $43.9(29.4)[1135]$ & $39.0(29.6)[1661]$ & $<0.01$ \\
\hline $\begin{array}{l}\text { Patient global assessment of arthritis (VAS } \\
0-100 \text { ) }\end{array}$ & $41.8(29.5)[1132]$ & $38.0(30.1)[1663]$ & $<0.01$ \\
\hline $\begin{array}{l}\text { Patient global assessment of arthritis and } \\
\text { psoriasis (VAS } 0-100 \text { ) }\end{array}$ & $41.6(29.0)[1130]$ & $37.4(30.0)$ [1659] & $<0.01$ \\
\hline Morning stiffness & & & \\
\hline & 380 & 600( & \\
\hline$\geq 30$ & & & \\
\hline $\mathrm{HAQ}(0-3)$ & $0.7(0.7)$ & $0.6(0$. & $<0.01$ \\
\hline EQ-5D & $0.7(0.2)$ & 0.8 & $<0.01$ \\
\hline $\operatorname{EQ~VAS~}(0-100)$ & $68.6(21.4)[1127]$ & $72.1(21.1)[1663]$ & $<0.01$ \\
\hline \multicolumn{4}{|l|}{ WPAI domains } \\
\hline Current employment, $\mathrm{n} / \mathrm{m}\left({ }^{\circ}\right.$ & & $36(61.3)$ & 0.93 \\
\hline me missed & 5.9 & 4.6 & 0.2 \\
\hline & & & \\
\hline$\%$ Overall work impairment & $23.2(26.1)[540]$ & $20.5(25.3)[839]$ & 0.02 \\
\hline \% Activity impairment & $32.5(30.2)[1026]$ & $26.6(28.0)[1543]$ & $<0.01$ \\
\hline \multicolumn{4}{|c|}{$\begin{array}{l}\text { EQ-5D, EuroQol } 5 \text { Dimensions questionnaire; } \mathrm{HAQ}-\mathrm{DI}, \text { Health Assessment Questionnaire Disability Index; } \\
\text { MDA, minimal disease activity; PSO, psoriasis; VAS, visual analog scale; WPAI, Work Productivity and } \\
\text { Activity Impairment questionnaire. } \\
\text { "All values were calculated based on available data and are presented as "mean (SD) [n]" unless otherwise } \\
\text { stated. } \\
t \text { t-test or Wilcoxon rank-sum test for continuous variables and } x^{2} \text { or Fisher's exact test for categorical } \\
\text { variables. }\end{array}$} \\
\hline
\end{tabular}

None declared, Blessing Dube Employee of: B. Dube is an employee of Corrona, LLC., Meghan Glynn Employee of: M. Glynn is an employee of Corrona, LLC., Alexis Ogdie Grant/research support from: (To my university) Novartis, Pfizer, Grant/research support from: Novartis, Pfizer, Grant/research support from: Novartis, Pfizer, Grant/research support from: Novartis, Pfizer, Consultant for: AbbVie, Bristol-Myers Squibb, Celgene, Corrona, Eli Lilly and Company, Novartis, Pfizer, and Takeda, Consultant for: AbbVie, Amgen, Bristol-Myers Squibb, Celgene, Corrona, Eli Lilly, Novartis, Pfizer Inc, Takeda, Consultant for: Abbvie, Amgen, BMS, Celgene, Corrona, Lilly, Novartis, Pfizer, Takeda, Consultant for: Abbvie, Amgen, BMS, Celgene, Corrona, Lilly, Novartis, Pfizer, Takeda

DOI: 10.1136/annrheumdis-2019-eular.1038

\section{SAT0389 INHIBITIONOF RADIOGRAPHIC PROGRESSION WAS ACHIEVED WITH INTRAVENOUS GOLIMUMAB IN ACTIVE PSA PATIENTS REGARDLESS OF CHANGES IN COMPOSITE INDICES OF DISEASE ACTIVITY IN A PHASE III TRIAL}

Philip J. Mease ${ }^{1}$, M Elaine Husni ${ }^{2}$, Shelly Kafka ${ }^{3}$, Soumya D. Chakravarty ${ }^{3,4}$, Diane D. Harrison ${ }^{5}$, Kim Hung Lo ${ }^{5}$, Stephen $\mathrm{Xu}^{5}$, Elizabeth C. Hsia, ${ }^{5,6}$,

Arthur Kavanaugh ${ }^{7} .{ }^{1}$ Swedish Medical Center and Univ of Wash School of Med, Seattle, United States of America; ${ }^{2}$ Cleveland Clinic, Cleveland, United States of America; ${ }^{3}$ Janssen Scientific Affairs, LLC, Horsham, United States of America; ${ }^{4}$ Drexel Univ College of Med, Phila, United States of America; ${ }^{5}$ Janssen Research and Development, LLC, Spring House, United States of America; ${ }^{6}$ Univ of Penn Medical Center, Phila, United States of America; ' Univ of CA San Diego, San Diego, United States of America

Background: GO-VIBRANT is a Phase 3 trial of intravenous (IV) golimumab (GLM), an anti-tumor necrosis factor alpha (TNF $\alpha$ ) monoclonal antibody, in adult patients (pts) with active psoriatic arthritis (PsA).

Objectives: To assess if changes in Disease Activity in PsA (DAPSA), PsA Activity Score (PASDAS), Minimal Disease Activity (MDA), Very Low Disease Activity (VLDA), and Clinical Disease Activity Index (CDAI) measures correlate with $\mathrm{X}$-ray progression.

Methods: In this multicenter, randomized, double-blind, placebo (PBO)controlled trial, 480 bionaïve PsA pts with active disease $(\geq 5$ swollen $\& \geq 5$ tender joints, C-reactive protein $\geq 0.6 \mathrm{mg} / \mathrm{dL}$, active plaque psoriasis or documented history despite treatment w/csDMARDs \&/or NSAIDs) received IV GLM $2 \mathrm{mg} / \mathrm{kg}(\mathrm{N}=241)$ at $W \mathrm{ks} 0 / 4$ then q8wks or PBO ( $\mathrm{N}=239)$ at $W k s 0 / 4 / 12 / 20$ with crossover to GLM at Wk24. In a post-hoc analysis, association of disease activity measures DAPSA, PASDAS, MDA, VLDA, \& CDAI with X-ray progression was examined. Total modified van der Heijde-Sharp ( $\mathrm{vdH}-\mathrm{S})$ score assessed X-ray progression at Wks 0/24/52. Last observation carried forward imputation was used for partially missing data \& non-responder imputation for missing data. Nominal $p$-values are reported without multiplicity adjustment.

Results: Mean changes from baseline in vdH-S scores were lower with GLM than PBO at Wk24 ( -0.36 vs 1.95 , respectively, $\mathrm{p}<0.001)$ and at Wk52 after crossover from PBO to GLM arm (-0.49 vs 0.76$)$. Changes in all disease activity measures appeared to correlate with X-ray progression (Table). GLM-treated pts had less X-ray progression regardless of disease activity measure. GLM treated pts in remission or with low disease activity tended to have less X-ray progression at Wk52 vs pts with moderate or high disease activity (mean change in vdH-S: DAPSA remission or low disease activity -0.88 , moderate activity -0.48 , high disease activity 0.41). Similar patterns were seen with PASDAS and CDAl (Table). Irrespective of level of disease activity, GLM-treated pts from Wk 0-52 tended to have less $\mathrm{X}$-ray progression vs PBOtreated pts who switched to GLM at Wk24 (mean change in vdH-S 052 wk GLM vs $\mathrm{PBO} \rightarrow \mathrm{GLM}:$ DAPSA remission or low disease activity 0.88 vs 1.49 , moderate activity -0.48 vs 1.38 , high disease activity 0.41 vs 1.27 )

Interestingly, pts treated with GLM who did not achieve MDA or VLDA by Wk52 also tended to have less X-ray progression vs PBO pts (mean change no MDA GLM 0.03 vs PBO 1.50; $\mathrm{p}=0.0011$ and mean change no VLDA GLM -0.30 vs PBO $1.45 ; p<0.0001)$.

Conclusion: In this analysis, all disease activity measures generally correlated with X-ray progression from baseline to Wk24 and to Wk52. Higher disease activity was associated with increased X-ray progression. GLMtreated pts not achieving MDA \& VLDA at Wk52 tended to have less $X$ ray progression vs $\mathrm{PBO} \rightarrow \mathrm{GLM}$ pts. GLM's ability to inhibit X-ray progression, despite pts not being in clinical remission or low disease activity, illustrates an example of "disconnect" between clinical outcomes \& X-ray progression seen in other studies.

Disclosure of Interests: Philip $J$ Mease Grant/research support from: AbbVie, Amgen, BMS, Celgene, Janssen, Lilly, Novartis, Pfizer, SUN and UCB, Consultant for: AbbVie, Amgen, BMS, Galapagos, Gilead Sciences, 